\title{
Novel Possibilities for the Diagnosis of Fetal Distress in Pre-eclamptic Women
}

\author{
Igor Victorovich Lakhno
}

\section{ABSTRACT}

Objective: Fetal deterioration has been known as a result of acute or chronic disturbances in the system of 'motherplacenta-fetus'. Pre-eclampsia (PE) is a maternal gestational disease caused by abnormal placentation that could provoke fetal distress.

Aim: The aim of the investigation was to survey the accuracy of fetal distress diagnostics in case of traditional cardiotocography (CTG) waveform evaluation and electrocardiography (ECG) parameters analysis obtained from maternal abdominal signal before and during labor in pre-eclamptic patients.

Materials and methods: One hundred and thirty-nine pregnant ladies at 32 to 40 weeks of gestation were enrolled in the study. There were 30 women with physiological pregnancy and normal fetal condition according to hemodynamic Doppler values and fetal ECG parameters in group I. In group II, 64 patients with mild and moderate PE were involved whom were performed fetal ECG noninvasive monitoring and hemodynamic Doppler. In group III, 45 patients with severe PE were monitored with fetal noninvasive ECG and hemodynamic Doppler.

Results: It was determined that fetal autonomic tone was suppressed with the relative increase of low frequency branch in pre-eclamptic patients. The increased value of the amplitude of mode and stress index was associated with abnormal myocardial adrenergic stimulation. It has induced $p Q$ and $Q T$ shortening, increased T/QRS ratio. Fetal cardiac response was determined as gradual reduce of autonomic regulation.

The most considerable negative correlation was determined in pair Apgar score 1 vs T/QRS $(R=-0.48 ; p<0.05)$. The QT interval duration has revealed positive correlation with Apgar score $1(R=0.44 ; p<0.05)$. Additional to the conventional CTG analysis evaluation of noninvasive fetal ECG parameters has contributed to better diagnostic of distress.

Conclusion: Fetal noninvasive ECG has showed sensitivity $100.0 \%$ and specificity $93.8 \%$ and, therefore, was more accurate and convenient method for fetal monitoring than conventional CTG.

Keywords: Fetal distress, Fetal noninvasive ECG monitoring, Pre-eclampsia.

\section{Associate Professor}

Department of Perinatology, Obstetrics and Gynecology Kharkiv Medical Academy of Postgraduate Education Kharkiv, Ukraine

Corresponding Author: Igor Victorovich Lakhno, Associate Professor, Department of Perinatology, Obstetrics and Gynecology, Kharkiv Medical Academy of Postgraduate Education, Kharkiv, Ukraine, Phone: 380577113556, e-mail: igorlakhno71@gmail.com
How to cite this article: Lakhno IV. Novel Possibilities for the Diagnosis of Fetal Distress in Pre-eclamptic Women. Int $\mathrm{J}$ Infertil Fetal Med 2015;6(2):62-66.

\section{Source of support: Nil}

Conflict of interest: None

Date of received: $18-06-15$

Date of acceptance: $22-07-15$

Date of publication: August 2015

\section{INTRODUCTION}

Fetal compromise has been known as a result of acute or chronic disturbances in the system of 'mother-placentafetus'. Fetal growth retardation is associated with chronic placental insufficiency and low Apgar score., ${ }^{1,2}$ Preeclampsia (PE) is a maternal gestational disease caused by abnormal placentation that may provoke fetal distress. ${ }^{3}$ The introduction of fetal heart rate electronic monitoring has contributed enormously to the advancement and evolution of fetal medicine. The well known approach is based on the registration of ultrasound waves reflected from fetal cardiac structures. Ultrasound cardiotocography (CTG) permits to register continual changes of the mechanically detected cardiointervals and estimate the fetal heart rhythm complexity.

Fetal heart rate variability (HRV) is a convenient tool for the fetal biophysical activity and neurodevelopmental investigations. ${ }^{4,5}$ But routine HRV parameters obtained with CTG are not absolutely specific for any fetal status within periods of its motile activity and quiet or active sleep. Fetal motile activity is associated with an increased total power (TP) spectrum of HRV and, therefore, with augmented low frequency (LF) and very low frequency (VLF) domain regions values. The power of LF and VLF branches indicates the central sympathetic and humoral vasoactive response on fetal activity. Very low frequency is traditionally associated with thermal regulation, the function of the hypothalamic-pituitary-adrenal axis and renin-angiotensin system activity. ${ }^{5}$ The periods of fetal respiratory movements are accompanied with high frequency (HF) domain region predominance. This peculiarity demonstrates the vagal nature of cardiac and respiratory synchronization. ${ }^{6}$ Fetal sleep has its suppressive influence on TP and all branches of HRV 
power spectrum and may produce a rigid (nonreactive) rhythm on CTG tracing. Therefore, the correction for sleep on antenatal CTG patterns is necessary. Otherwise, the optimization of CTG outcome requires prolonged period of registration even for several hours. It is not acceptable in the real urgent obstetrical situation at decision. Fetal neurobehavioral response stimulates the search for novel technological approaches of its assessment.

The detection of the primary bioelectrical processes obtained from maternal abdominal wall with non-invasive electrocardiography (ECG) could provide the higher precision of CTG waveform. Fetal HRV parameters have a very wide range even in normal condition. It may complicate the interpretation and increase the level of unfounded cesarean deliveries. ${ }^{3-6}$ Additional investigation of the fetal ECG morphological parameters could contribute to better diagnostics of its functional status. It has been revealed that increased T/QRS ratio due to elevated T-wave was a marker of the fetal distress. ${ }^{7-11}$ The activation of anerobic processes in myocardium has been reflected in T-wave elevation. Recent investigations have invented that fetal hypoxia could cause the shortening of QT interval. ${ }^{9}$ But fetal noninvasive ECG expectations are not still absolutely clear. High signal to noise ratio complicates wide introduction of the method. The most considerable databases of fetal ECG morphological parameters were obtained with usage of magnetocardiography. ${ }^{11}$ This method requires high expenses and not in routine use now. Fetal noninvasive ECG could provide more evidence in case of umbilical blood flow abnormalities and fetal immaturity, suspected false-negative nonstress test or intrapartum decelerations. The maximal accuracy in fetal status assessment could decrease obstetrical aggression. That is why fetal noninvasive ECG could become a prospective supplementation to traditional CTG, fetal biophysical profile and Doppler ultrasonography.

The ukrainian scientists have developed 'Cardiolab Babycard' fetal noninvasive ECG monitor lately. The ECG recordings obtained in Ukraine were included in PhysioNet database. ${ }^{12}$

The investigation was aimed to survey the accuracy of fetal distress diagnostics in case of traditional CTG waveform evaluation and ECG parameters analysis obtained from maternal abdominal signal before and during labor in pre-eclamptic patients.

\section{MATERIALS AND METHODS}

The study protocol was approved by the bioethics committee of the medical academy of postgraduate education. Observed pregnant ladies were informed about the methods of the study, its aim, indications and eventual complications before inclusion in the study. All patients gave written informed consent to participate in the investigation.

The cross-sectional study was conducted from May 2012 to August 2014.

\section{Inclusion Criteria}

Diagnosed PE based on blood pressure more than 140/90 $\mathrm{mm} \mathrm{Hg}$ on two separate occasions 6 hours apart, positive proteinuria test in two mildstream urine samples collected 4 hours apart.

\section{Exclusion Criteria}

Multiple pregnancy, eclampsia, pre-existing medical disorders, like diabetes mellitus, cardiac diseases, renal disease, thyrotoxycosis and chronic hypertension without superimposed PE.

One hundred and thirty-nine pregnant ladies at 32 to 40 weeks of gestation were enrolled in the study. Patients were divided into three groups randomly. There were 30 women with physiological pregnancy and normal fetal condition according to hemodynamic Doppler values and fetal ECG parameters in group I. In group II, 64 patients with mild and moderate PE were involved whom were performed fetal ECG noninvasive monitoring and hemodynamic Doppler. In group III, 45 patients with severe PE were monitored with fetal non-invasive ECG and hemodynamic Doppler. Fetal non-invasive ECG monitoring was continued in labor.

All observed preeclamptic women have received antihypertensive drugs according to the type of central maternal hemodynamics determined with bioimpedance cardiography. The pregnant patients with hyperkinetic type of central maternal hemodynamic have taken carvedilol at 6.25 to $12.5 \mathrm{mg} 2$ times daily, in case of eukinetic type-methyldopa 250 to $500 \mathrm{mg} 4$ times a day and at hypokinetic one-methyldopa $500 \mathrm{mg} 4$ times daily combined with nifedipine $20 \mathrm{mg} 2$ times day.

Fetal noninvasive ECG parameters were obtained with the application of the computer electrocardiographic system 'Cardiolab Baby Card' (Kharkiv, Ukraine). The registration was carried out for 10 minutes long. It was estimated the value of TP and its spectral compounds: VLF, LF and HF. The temporal characteristics of the fetal HRV: standard deviation of normal to normal intervals $(\mathrm{SDNN})$, root mean square of successive differences (RMSSD), the proportion of the number of pairs of successive NNs that differ by more than 50 ms divided by total number of NNs (pNN50), the number of cardiointervals, corresponding to the value of mode (or within modal interval) in \% of the volume of the sample-the amplitude of mode (AMo) and the ratio of the height of the histogram to its width that characterized the degree 
of tension of regulatory systems-stress index (SI) were determined. The CTG waveform analysis was performed with short-term variability (STV) and long-term variability (LTV) scoring. The fetal ECG parameters, such as $\mathrm{PQ}, \mathrm{QT}$ intervals and QRS complex duration, T-wave amplitude and T/QRS ratio were calculated.

It was investigated the share of instrumentally determined fetal distress in antenatal period and Apgar score 1 after delivery.

The results were processed by parametric statistical methods (mean-M, error $-\mathrm{m}$ ) with the application of statistics software package Excel adapted for biomedical research. The results, thus, obtained were subjected to standard statistical analysis and analyzed using the Chi-square test to compare for categorical data between the groups. The significance was set at $\mathrm{p}$-value $<0.05$. For the statistical analysis of relationship between $X$ and $\mathrm{Y}$, correlations coefficients were estimated using Spearman's test. The sensitivity and specificity of diagnostic tests were calculated.

\section{RESULTS}

The obtained data have demonstrated the significant difference in fetal HRV parameters in the observed groups (Table 1). It was determined the decrease in TP and SDNN values proportionally to the severity degree of PE but mostly in group III. Fetal autonomic tone was lower with relative increase of LF domain region in all patients with PE. The increased values of AMo and SI in the group II and group III were associated with abnormal myocardial adrenergic stimulation and had reflected the stressed metabolic status. The mean values of short-term mediated parameters: RMSSD, pNN5O, HF and STV were significantly decreased in PE. It was revealed the predominance of central circuit activity over autonomic one in the fetuses of pre-eclamptic women. The suppression of respiratory sinus arrhythmia was the predisposing condition to the loss of nonlinearity in cardiac activity. So, fetal hypersympatheticotonia was one of the main events in PE-induced scenario.

The progradient growth of the autonomic (LF/HF) balance was determined in groups I, II and III (Graph 1). These transformations have been associated with fetal hypersympatheticotonia in pre-eclamptic patients.

The evolution of the fetal ECG peaks and intervals parameters in PE has demonstrated gradual response to sympathetic hyperstimulation (Table 2). In case of mild and moderate PE, the tendency to tachycardia was associated with prolonged $\mathrm{pQ}$ value. The decrease of mean fetal heart rate and predominant decelerative pattern of CTG waveform in the group III were accompanied with significant $\mathrm{pQ}$ shortening. So, the lowered $\mathrm{pQ}$ was a mirror of decelerations. The elevation of T-wave has reflected in the increased T/QRS ratio. It was revealed the decreased duration of QT interval. The affected myocardium has responded high $\mathrm{T} / \mathrm{QRS}$ ratio.

The investigation of statistically significant correlations between newborn Apgar score 1 and fetal HRV, CTG and noninvasive ECG parameters has revealed certain regularities (Graph 2). The most considerable negative correlation was determined in pair Apgar score 1 versus $\mathrm{T} / \mathrm{QRS}(\mathrm{R}=-0.48 ; \mathrm{p}<0.05)$. So, the increased T/QRS ratio was the most evident marker of fetal distress. The QT interval duration has revealed positive correlation with Apgar score $1(\mathrm{R}=0.44 ; \mathrm{p}<0.05)$. The validity of fetal HRV parameters was quite different. The negative correlation was determined between Apgar score 1 vs SI $(\mathrm{R}=-0.40$; $\mathrm{p}<0.05)$ and Apgar score 1 vs AMo $(\mathrm{R}=-0.39 ; \mathrm{p}<0.05)$. It was not determined a considerable relationship between SDNN vs Apgar score $1(\mathrm{R}=0.20 ; \mathrm{p}<0.05)$ and $\mathrm{TP}$ vs

Table 1: Fetal HRV parameters in observed ladies

\begin{tabular}{|c|c|c|c|}
\hline Index, units of measure & Group I & Group II & Group III \\
\hline SDNN, ms & $50.3 \pm 11.0$ & $47.4 \pm 9.5^{*}$ & $24.6 \pm 8.8^{* / * *}$ \\
\hline RMSSD, ms & $22.4 \pm 9.6$ & $19.2 \pm 7.2^{*}$ & $7.9 \pm 2.5^{\star} / * *$ \\
\hline pNN5O, \% & $8.4 \pm 2.1$ & $7.6 \pm 2.0^{*}$ & $3.3 \pm 0.5^{\star / * *}$ \\
\hline SI, conv. units & $241.6 \pm 22.8$ & $384.2 \pm 52.4^{*}$ & $1457.2 \pm 345.0^{*} /$ ** \\
\hline AMo, $\%$ & $38.0 \pm 10.4$ & $43.6 \pm 11.4^{*}$ & $62.5 \pm 17.6^{\star / * \star}$ \\
\hline $\mathrm{TP}, \mathrm{ms}^{2}$ & $1635 \pm 364.2$ & $1443.4 \pm 299.5^{\star}$ & $530.1 \pm 145.4^{*} / * *$ \\
\hline $\mathrm{VLF}, \mathrm{ms}^{2}$ & $1351.2 \pm 384.5$ & $996.2 \pm 84.6^{*}$ & $345.2 \pm 23.8^{* / *}$ \\
\hline $\mathrm{LF}, \mathrm{ms}^{2}$ & $189.6 \pm 31.1$ & $317.2 \pm 66.8^{*}$ & $148.6 \pm 25.1^{\star / * *}$ \\
\hline $\mathrm{HF}, \mathrm{ms}^{2}$ & $95.2 \pm 19.4$ & $132.1 \pm 34.5^{\star}$ & $37.2 \pm 14.6^{\star / * \star}$ \\
\hline STV, ms & $8.8 \pm 2.5$ & $6.0 \pm 2.4^{*}$ & $3.8 \pm 1.5^{* / *}$ \\
\hline LTV, ms & $38.5 \pm 14.1$ & $31.4 \pm 9.3^{*}$ & $17.2 \pm 6.8^{* / * *}$ \\
\hline
\end{tabular}

*The differences were statistically significant compared to the control group $(p<0,05)$; ${ }^{* *}$ the differences were statistically significant compared to the group II $(p<0,05)$; SDNN: standard deviation of normal to normal intervals; RMSSD: root mean square of successive differences; pNN50: the proportion of the number of pairs of successive NNs that differ by more than 50 ms divided by total number of NNs; AMo: the number of cardiointervals, corresponding to the value of mode (or within modal interval) in \% of the volume of the sample; SI: the ratio of the height of the histogram to its width that characterized the degree of tension of regulatory systems; TP: total power; VLF: very low frequency; LF: low frequency; HF: high frequency; STV: short-term variability; LTV: long-term variability 


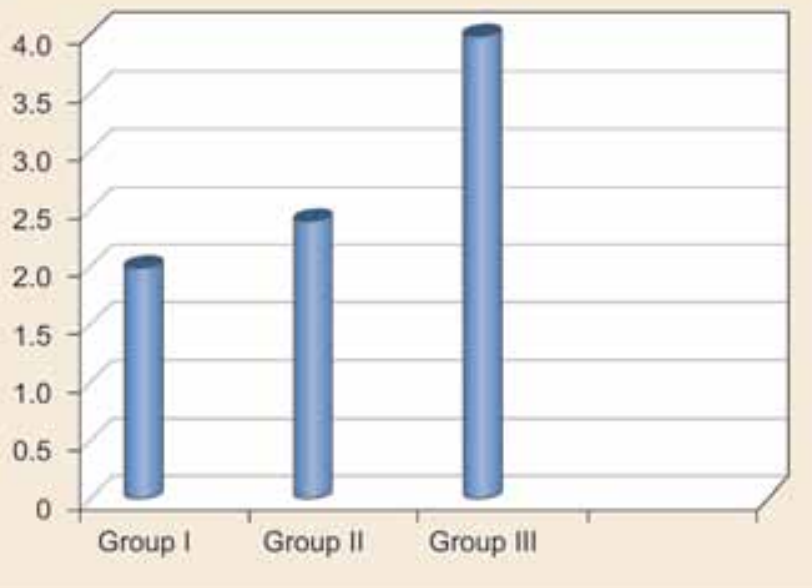

Graph 1: Fetal autonomic balance in observed ladies

Table 2: Fetal ECG parameters in observed ladies

\begin{tabular}{llll}
\hline $\begin{array}{l}\text { Index, units of } \\
\text { measure }\end{array}$ & \multicolumn{1}{c}{ Group I } & \multicolumn{1}{c}{ Group II } & \multicolumn{1}{c}{ Group III } \\
\hline $\mathrm{pQ}, \mathrm{ms}$ & $101.5 \pm 19.0$ & $106.2 \pm 14.3^{*}$ & $95.6 \pm 18.7^{*} /^{* *}$ \\
$\mathrm{QT}, \mathrm{ms}$ & $222.5 \pm 28.6$ & $209.2 \pm 25.1^{*}$ & $194.8 \pm 31.3^{* / *}$ \\
$\mathrm{QRS}, \mathrm{ms}$ & $64.3 \pm 12.3$ & $64.5 \pm 10.4$ & $65.7 \pm 11.0^{* / * *}$ \\
$\mathrm{~T}, \mathrm{mcV}$ & $2.8 \pm 0.5$ & $4.2 \pm 1.1^{*}$ & $8.2 \pm 2.1^{*} /^{* *}$ \\
$\mathrm{~T} / \mathrm{QRS}$ & $0.04 \pm 0.01$ & $0.08 \pm 0.03^{*}$ & $0.20 \pm 0.05^{* / *}$ \\
\hline
\end{tabular}

${ }^{*}$ The differences were statistically significant compared to the control group $(p<0.05)$; ${ }^{*}$ The differences were statistically significant compared to the group II $(p<0.05)$

Apgar score 1 ( $\mathrm{R}=0.22 ; \mathrm{p}<0.05)$. It was confirmed that autonomic tone has a weak connection with fetal distress. Fetal vagal tone has revealed a correlation with Apgar score $1(\mathrm{R}=0.35 ; \mathrm{p}<0.05)$. The negative correlation between fetal sympathetic tone and Apgar score 1 has been determined $(\mathrm{R}=-0.28 ; \mathrm{p}<0.05)$. The parameters of CTG has demonstrated weak correlation with Apgar score 1: STV $(R=0.24 ; p<0.05)$ and LTV $(R=0.18 ; p<0.05)$. The obtained data has confirmed speculation that fetal noninvasive ECG parameters have considerable significance in fetal distress diagnostics.

\section{DISCUSSION}

Fetal neurodevelopmental status in PE was associated with central sympathetic activation in its projections on hemodynamics. Fetal cardiac response has been determined as gradual reduce of autonomic regulation. ${ }^{13}$ But it has been revealed lack correlation between sympathetic tone and Apgar score 1. So, hyperactivity of sympathetic regulation was found as partially adaptive reaction on abnormal hemodynamics in fetoplacental system. Excessive activity of the sympathetic division of the autonomic nervous regulation have induced redundant baroreflex response. It was performed the speculation that the origin of the antenatal decelerations was presented in the pronounced augmentation of the sympathetic baroreflex.

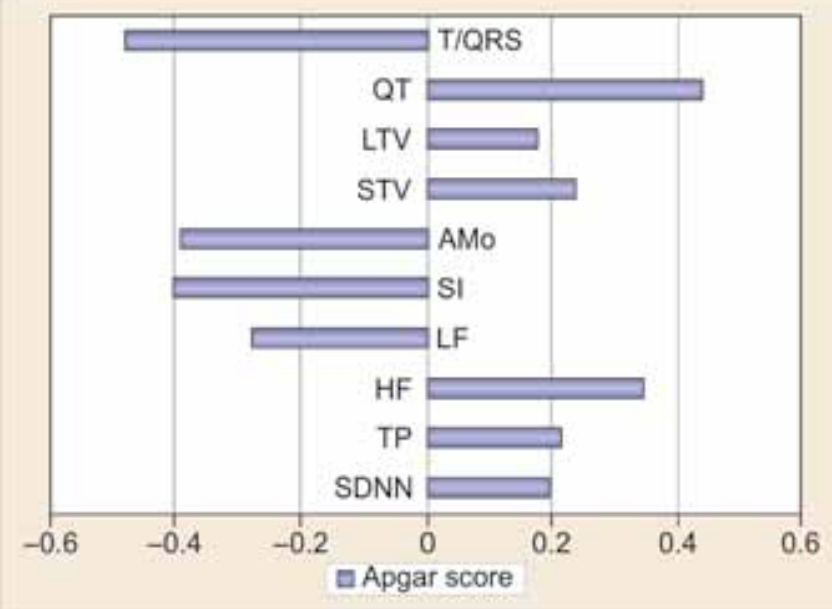

Graph 2: Correlations between Apgar score 1 and values of HRV, CTG and morphological ECG parameters in the study population

The decrease in vagal regulation and hypothalamic-pituitary-adrenal axis function failure were also involved in the fetal compromise. ${ }^{1,2,5}$ Mutual activation of vagal and sympathetic branches of autonomic function may provoke fetal aspiration. Fetal intestinal hypermotility and meconium passage based on increased catecholamines level accompanied by respiratory activity could induce intrauterine aspiration. But this speculation requires further investigations.

The obtained data have highlighted the use of SI and AMo as universal markers of fetal hypersympatheticotonia. It has been revealed that SI and AMo values were independent from fetal stationary condition and sensitive for fetal distress even in labor. But fetal noninvasive ECG morphological parameters have demonstrated best significance in fetal distress diagnostics. The decreased QT duration and the rise of $\mathrm{T}$ wave amplitude were the symptoms of $\beta$-adrenergic receptors activation.-11 Increased T/QRS ratio has been known as more valid marker of fetal distress in several studies..$^{8,10,11,14}$ The presence of decelerations on CTG tracing as a response to fetal motile activity before labor or uterine contractions in labor makes obstetrician to think about fetal distress. Additional investigation of QT interval or T/QRS ratio could confirm or neglect this diagnosis.

The rate of fetal distress retrospectively was $33.0 \%$ in PE. This result was rather logical. ${ }^{1,3,13}$ But additional to the conventional CTG analysis evaluation of noninvasive ECG parameters has contributed to better diagnostic of fetal distress. The values of sensitivity and specificity of noninvasive fetal ECG were 100.0 and $93.8 \%$ respectively. The traditional analysis of CTG parameters has showed sensitivity $94.4 \%$ and specificity $83.3 \%$.

Fetal noninvasive ECG has showed additional possibilities to conventional CTG. This type of fetal monitoring is more preferable in prediction of perinatal outcome. 
Further wide introduction of fetal noninvasive ECG could avoid obstetrical aggression and unfounded interventions. The possibilities of continual wireless remote fetal monitoring offers novel prospects in perinatology.

\section{CONCLUSION}

Fetal noninvasive ECG has showed sensitivity 100\% and specificity $93.8 \%$ and, therefore, was more accurate and convenient method for fetal monitoring than conventional CTG.

\section{REFERENCES}

1. Graatsma EM, Mulder EJH, B Vasak B, et al. Average acceleration and deceleration capacity of the fetal heart rate in normal pregnancy and in pregnancies complicated by fetal growth restriction. J Matern Fetal Neonatal Med 2012;25(12):2517-2522.

2. Aziz W, Schlindwein FS, Wailoo M, et al. Heart rate variability analysis of normal and growth restricted children. Clin Auton Res 2012;22(2):91-97.

3. Hladunewich M, Karumanchi SA, Lafayette R. Pathophysiology of the clinical manifestations of preeclampsia. Clin J Am Soc Nephrol 2007;2(3):543-549.

4. Brown CA, Lee CT, Hains SM, Kisilevsky BS. Maternal heart rate variability and fetal behavior in hypertensive and normotensive pregnancies. Biol Res Nurs 2008;10(2):134-144.

5. David M, Hirsch M, Karin J, et al. An estimate of fetal autonomic state by time-frequency analysis of fetal heart rate variability. J Appl Physiol 2007;102(3):1057-1064.
6. Ortiz MR, Echeverria JC, Alvarez-Ramirez J, et al. Effects of fetal respiratory movements on the short-term fractal properties of heart rate variability. Med Biol Eng Comput 2013;51(4):441-448.

7. Clifford G, Sameni R, Ward J, et al. Clinically accurate fetal ECG parameters acquired from maternal abdominal sensors. Am J Obstet Gynecol 2011;205(1):47.e1-47.e5.

8. Karvounis EC, Tsipouras MG, Papaloukas C, et al. A noninvasive methodology for fetal monitoring during pregnancy. Methods Inf Med 2010;49(3):238-253.

9. Oudijk MA, Kwee A, Visser GHA, et al. The effects of intrapartum hypoxia on the fetal QT interval. BJOG: an Int J Obstet Gynaecol 2004;111(7):656-660.

10. Rzepka R, Torbe A, Kwiatkowski S, et al. Clinical outcomes of high-risk labours monitored using fetal electrocardiography. Ann Acad Med Singapore 2010;39(1):27-32.

11. Van Leeuwen P, Lange S, Klein A, et al. Dependency of magnetocardiographically determined fetal cardiac time intervals on gestational age, gender and postnatal biometrics in healthy pregnancies. BMC Pregnancy Childbirth 2004; 4(1):6-16.

12. Silva I, Behar J, Sameni R, et al. Noninvasive fetal ECG: the Physio net/computing in cardiology challenge 2013/Comput Cardiol 2010;40:149-152.

13. Swansburg ML, Brown CA, Hains SM, et al. Maternal cardiac autonomic function and fetal heart rate in preeclamptic compared to normotensive pregnancies. Can J Cardiovasc Nurs 2005;15(3):42-52.

14. Reinhard J, Hayes-Gill BR, Yi Q, et al. Comparison of noninvasive fetal electrocardiogram to Doppler cardiotocogram during the 1st stage of labor. J Perinat Med 2010;38(2):179-185. 